\section{Fine Structure of Nebular Emission Lines}

THe fact that nebular lines may have significant fine structure seems to have been noglected by nebular spectroscopists who have been considering the turbulent and thermal broadening of these lines. For example, Aller ${ }^{1}$ and Courtès et al. ${ }^{2}$, who have measured or interpreted the widths of the lines, do not mention this effoct.

In particular $\mathrm{H} \alpha$ is composed of four components. Kent et al. ${ }^{3}$, Lewis and Spedding ${ }^{4}$ and Spedding et $a l .{ }^{5}$ showed experimentally, for a laboratory source, that only the two principal components, of nearly equal brightness, were significant. They are separated by $0 \cdot 14 \AA$. Sommerfeld and Unsöld ${ }^{6,7}$ and Schrödinger ${ }^{8}$ calculated theoretically the relative brightnesses of this fine structure. These depend strongly on the conditions of excitation.

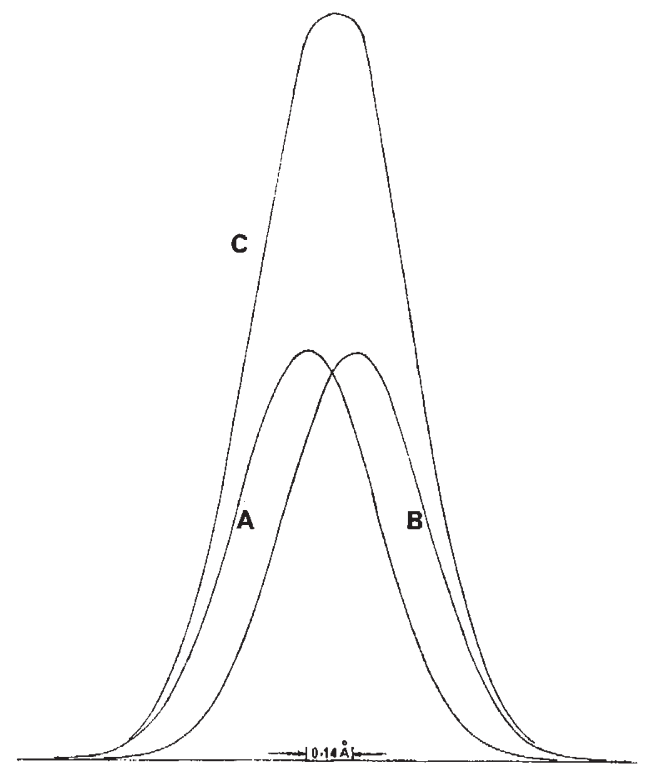

Fig. 1. 'Thermally' broadened component of the $\mathrm{H} \alpha$ line composed of two Gaussians A and B.

It is interesting to estimate the error introduced into the measurements of the electron temperatures of HII regions from the width of the $\mathrm{H} \alpha$ line if the fine structure is neglected. Consider the case shown in Fig. 1 where the thermally broadened component of the $H \alpha$ line is composed of two Gaussians A and B of equal brightness, where each has a thermally broadened width of $0.506 \AA$ corresponding to a nebular temperature of $10,000 \mathrm{~K}$ and where these two components are separated by $0 \cdot 14 \AA$. The thermally broadened component of the $\mathrm{H} \alpha$ line would then appear as the summation, C, of these two curves. This has a halfwidth of $0.545 \AA$. If this wore inadvertently considered to be a single Gaussian representing the thermal component of the $\mathrm{H} \alpha$ line, then the clectron temperature would be given wrongly as $11,880 \mathrm{~K}$. This indicates an error of around $+2,000 \mathrm{~K}$. It is then possible that every measurement of temperature of gaseous nebulae that has relied on the width of the $\mathrm{H} \alpha$ line and which has neglected this splitting is then 2,000 $\mathrm{K}$ overestimated. Those methods that depend on the widths of [NII] and $\mathrm{H} \alpha$ (ref. 2) are even further uncertain if the fine structure of this forbidden lino is also neglected.

It would seem necessary to eonsider the fine structure of all the nebular lines in the conditions existing in nebulae when making exact measurements of their widths and shapes.

Department of Astronomy,

J. Meaburn

University of Manchester.
Recelved August 23, 1970.

${ }^{1}$ Aller, L. H., International Astrophysics Series, 3, Gaseous Nebulae (Chapman and Hall Ltd, London, 1954).

${ }^{2}$ Courtès, G., Louise, R., and Monnet, G., Ann. l'A strophys., 31, 493 (1968).

${ }^{3}$ Kent, N. A., Tayler, L. B., and Pearson, H., Phys. Rev., 30, 266 (1927).

${ }^{4}$ Tewis, G. N., and Spedding, F. H., Phys. Rev., 43, 964 (1933).

Spedding, F. H., Shane, C. D., and Grace, N. S., Phys. Rev., 44, 58 (1933)

"Sommerfeld, A., and Unsöld, A., Zeit. f. Phys., 36, 259 (1926).

' Sommerfeld, A., and Unsöld, A., Zeit.f. Phys., 38, 237 (1926).

${ }^{8}$ Schrödinger, E., Ann. Phys., 80, 437 (1926).

\section{Apex of Solar Motion of Neutral Hydrogen Concentrations at Different Velocities}

De Vaucouleurs and Peters', Kerr and Sullivan² and $I^{3}$ derived the solar motion with respect to the high velocity $\mathrm{HI}$ clouds (HVCs) with a view to examining the suggestion by Verschuur that these cloud complexes and the Local Group of galaxies form part of the same extragalactic system of objects. They concluded that the solar motion solution does not seem to justify Verschuur's hypothesis. Kerr and Sullivan ${ }^{2}$ also derived the solar motion with respect to a sample of 125 intermediate velocity clouds (IVCs) and concluded that they seem to be kinematically distinct from the HVCs.

Dieter ${ }^{5}$ used the data from the Berkeley neutral hydrogen survey $\left(2 \mathrm{~km} \mathrm{~s}^{-1}\right.$ velocity resolution) to show that in almost every case high velocity condensations occur where there is evidence of excess gas at more moderate velocities. Using these data she also found evidence for physical interaction between gas masses at different velocities at the same location in the sky. This relation between gas at different velocities was based on her study of the velocity dispersion of the IVCs associated with the HVCs and also on the column densities of neutral hydrogen atoms.

In order to see if her data, which showed a physical relation between gas masses at different velocities, also showed a kinematic relationship, I divided her list (Table 1 of ref. 5) of 139 hydrogen condensations into two groups(1) 68 with velocities greater than $70 \mathrm{~km} \mathrm{~s}^{-1}$ and (2) 71 with velocities less than $70 \mathrm{~km} \mathrm{~s}^{-1}$-and derived the solar motion with respect to the local standard of rest separately for the two groups. The solutions are shown in Table 1. These results may be compared with those derived by de Vaucouleurs and Peters ${ }^{1}$, Kerr and Sullivan ${ }^{2}$ and $\mathrm{me}^{3}$ (Table 2).

Table 1. SOLAR MOTION SOLUTIONS RELATIVE TO LOCAI, STANDARD OF' RFST

$$
\begin{array}{ccccc}
\text { Group } & N & l_{\odot}^{\mathrm{II}} & b_{\odot}^{\mathrm{H}} & \mathrm{y} \mathrm{hm} \mathrm{s}^{-1} \\
>70 \mathrm{~km} \mathrm{~s}^{-1} & 68 & 129^{\circ} \pm 4^{\circ} & -12^{\circ} \pm 3^{\circ} & 27 \%-16 \\
<70 \mathrm{~km} \mathrm{~s}^{-1} & 71 & 148^{\circ} \pm 13^{\circ} & -12^{\circ} \pm 12^{\circ} & 91-9
\end{array}
$$

Table 2. RESULTS OF EARLIER WORK

$\left\{\begin{array}{rlll}N & l^{\mathrm{II}} & b_{\odot}^{\mathrm{rI}} & V_{0} \mathrm{~km} \mathrm{~s}^{-1} \\ 17 & 139^{\circ} \pm 16^{\circ} & -13^{\circ} \pm 13^{\circ} & 184 \pm 66 \\ 16 & 148^{\circ} \pm 7^{\circ} & -12^{\circ} \pm 5 \circ^{\circ} & 234 \pm 29 \\ 17 & 139^{\circ} \pm 20^{\circ} & -13^{\circ} \pm 16^{\circ} & 184 \pm 66 \\ 62 & 136^{\circ} & -44^{\circ} & 222 \pm 19 \\ 125 & 138^{\circ} & +42^{\circ} & 58 \pm 3\end{array}\right.$

Korr and Sullivan's solution for IVCs is different from my solution for gas at less than $70 \mathrm{~km} \mathrm{~s}^{-1}$. This may be due to the inclusion in their sample of 125 of $\mathrm{a}_{4}$ large proportion of IVCs that are not associated with HVCs.

It is seen from my solution (Table 1 ) that the apex of solar motion of gas with velocities greater than $70 \mathrm{~km} \mathrm{~s}^{-1}$ is not very different from that for gas with velocities less than $70 \mathrm{~km} \mathrm{~s}^{-1}$. The apices are in the galactic plane which seems to suggest that the gas concentrations are related to the galaxy. The close agreement between the dircetions of the solar motion of the two groups suggests that they 